Y. Furuta

Nagoya Math. J.

Vol. 93 (1984), 61-69

\title{
A NORM RESIDUE MAP FOR CENTRAL EXTENSIONS OF AN ALGEBRAIC NUMBER FIELD
}

\author{
YOSHIOMI FURUTA
}

Let $K$ be a finite Galois extension of an algebraic number field $k$ with $G=\operatorname{Gal}(K / k)$, and $M$ be a Galois extension of $k$ containing $K$. We denote by $K_{M / k}^{*}$ resp. $\hat{K}_{M / k}$ the genus field resp. the central class field of $K$ with respect to $M / k$. By definition, the field $K_{M / k}^{*}$ is the composite of $K$ and the maximal abelian extension over $k$ contained in $M$. The field $\hat{K}_{M / k}$ is the maximal Galois extension of $k$ contained in $M$ satisfying the condition that the Galois group over $K$ is contained in the center of that over $k$. Then it is well known that $\operatorname{Gal}\left(\hat{K}_{M / k} / K_{M / k}^{*}\right)$ is isomorphic to a factor group of the Schur multiplicator $H^{-3}(G, Z)$, and is isomorphic to $H^{-3}(G, Z)$ when $M$ is sufficiently large. In this case we call $M$ abundant for $K / k$ (See Heider [3, §4] and Miyake [6, Theorem 5]).

Let $G$ be abelian with a decomposition $G=G_{1} \times \cdots \times G_{r}$ to cyclic factors such that the order of $G_{i}$ is divisible by that of $G_{j}$ for $i<j$. Then the Schur multiplicator $H^{-3}(G, Z)$ is isomorphic to the second exterior power of $G$, and hence isomorphic to $\oplus \sum_{i<j} G_{j}$.

Corresponded with the above decomposition of $H^{-3}(G, Z)$, we show in Section 3 that the central class field $\hat{K}_{M / k}$ is the composite of central class fields over bicyclic subextensions of $K / k$ when $K$ is abelian over $k$ and $M$ is abundant for $K / k$ (Proposition 5). Then in Section 4 we define a mapping $\Psi_{M / K / k}$ via Artin's reciprocity map, which is a surjective homomorphism from a group of certain ideals of $k$ to $\oplus \sum_{i<j} G_{j} \cong \Lambda(G)$ (Theorem). The mapping $\Psi_{M / K / k}$ describes the prime decomposition in $\hat{K}_{M / k} / K_{M / k}^{*}$. On the other hand, in Section 2 we define a surjective homomorphism $\varphi_{M / K / k}$ from $\Lambda(G)$ to $\mathrm{Gal}\left(\hat{K}_{M / k} / K_{M / k}^{*}\right)$ by means of canonical cocycles of class field theory. The mapping $\Psi_{M / K / k}$ is regarded as the inverse of $\varphi_{M / K / k}$.

When $K$ is bicyclic biquadratic over the rational number field, the

Received September 14, 1982.

Revised February 21, 1983. 
mapping $\Psi$ is given explicitly in [2] by using solutions of certain quadratic diophantine equations.

\section{§1. Commutator factors of group extensions}

Let $G$ be a finite abelian group, and \&s be a group extension of an abelian group $A$ by $G$ : \& $/ A \cong G$. Let $\left\{U_{\sigma}\right\}$ be a system of representatives of $G$ in (S), and $\left\{C_{\sigma, \tau}\right\}$ be the factor system: $U_{\sigma} U_{\tau}=U_{\sigma \tau} C_{\sigma, \tau}$ for $\sigma, \tau \in G$. Denote by $I_{G}$ the augmentation ideal of the group ring $Z[G]$. Denote further by $\Lambda(G)$ the second exteror power of $G$. Then it is well known that $H^{-3}(G, Z) \cong \Lambda(G)$ (See, for instance, Razar [7, Lemma 5]). The following fact is also probably well known, but we prove it here because it is fundamental in this paper.

Proposition 1. Let $A, G$ and \&s be as above, and for $\sigma \wedge \tau \in \Lambda(G)$ let

$$
\varphi(\sigma \wedge \tau) \equiv C_{\sigma, \tau} C_{\tau, \sigma}^{-1} \quad \bmod I_{G} A .
$$

Then $\varphi$ induces a surjective homomorphism of $\Lambda(G)$ to $\left[\&,(ङ] / I_{G} A\right.$, where [\&, (ङ) is the commutator subgroup of \&s.

Proof. Let $a, b \in A$ and $\sigma, \tau \in G$. Then since $A$ and $G$ are abelian, we have $\left(U_{\sigma} a\right)^{-1}\left(U_{\imath} b\right)^{-1}\left(U_{\sigma} a\right)\left(U_{\tau} b\right) \equiv C_{\sigma, \tau} C_{\tau, \sigma}^{-1} a^{\tau-1}\left(b^{\sigma-1}\right)^{-1} \equiv C_{\sigma, \tau} C_{\tau, \sigma}^{-1} \bmod I_{G} A$. Hence if we put $\varphi_{1}(\sigma, \tau)=C_{\sigma, \tau} C_{\tau, \sigma}^{-1} \bmod I_{G} A, \varphi_{1}$ defines a mapping of $G \times G$

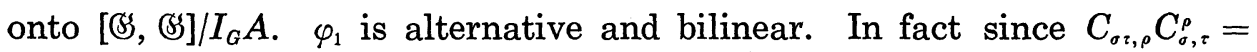
$C_{\sigma, \tau \rho} C_{\tau, \rho}$ for any $\sigma, \tau, \rho \in G$, we have

$$
\begin{aligned}
\frac{C_{\sigma \tau, \rho}}{C_{\sigma, \rho} C_{\tau, \rho}} & =\frac{C_{\sigma, \tau \rho}}{C_{\sigma, \tau}^{\rho} C_{\sigma, \rho}}=\frac{C_{\sigma, \tau \rho}}{C_{\sigma, \tau} C_{\sigma, \rho}} \cdot C_{\sigma, \tau}^{1-\rho} \\
& \equiv \frac{C_{\sigma, \tau \rho}}{C_{\sigma, \tau} C_{\sigma, \rho}} \quad \bmod I_{G} A .
\end{aligned}
$$

The first form is symmetric for $\sigma$ and $\tau$, and the last form is so for $\tau$ and $\rho$, because $G$ is abelian. Hence we have

$$
\frac{C_{\sigma \rho, \tau}}{C_{\sigma, \tau} C_{\rho, \tau}} \equiv \frac{C_{\tau, \sigma \rho}}{C_{\tau, \sigma} C_{\tau, \rho}} \quad \bmod I_{G} A
$$

This implies that $\varphi_{1}$ is alternative bilinear map, and the proposition is implied.

Remark. It is easy to see that $\varphi$ does not depend on the choice of the factor system $\left\{C_{\sigma, \tau}\right\}$. 


\section{§2. Mapping $\varphi_{M / K / k}$}

We apply Proposition 1 to an abelian Galois tower $M / K / k$, which means that both $M / K$ and $K / k$ are abelian extensions and $M / k$ is a Galois extension of algebraic number fields. Put $\mathbb{S}=\mathrm{Gal}(M / k), A=\mathrm{Gal}(M / K)$ and $G=\operatorname{Gal}(K / k)$. Then the canonical cocycle $\xi_{K / k}$ of class field theory gives a factor system for $\mathbb{S} / A \cong G$. For any algebraic number field $L$, we denote by $J_{L}$ the idele group of $L$, and by $L^{\times}$the group of principal ideles of $L$. Denote further by $H\left(L^{\prime} / L\right)$ the subgroup of $J_{L}$ corresponding to $L^{\prime}$ by class field theory when $L^{\prime}$ is a Galois extension of $L: H\left(L^{\prime} / L\right)=$ $L^{\times} \cdot N_{L, / L} J_{L}$.

Now we define a mapping $\varphi_{M / K / k}$ of the second exterior power $\Lambda(G)$ of $G$ to $J_{k} / H\left(\hat{K}_{M / k} / K\right)$ by

$$
\varphi_{M / K / k}(\sigma \wedge \tau) \equiv \xi_{K / k}(\sigma, \tau) \xi_{K / k}(\tau, \sigma)^{-1} \quad \bmod H\left(\hat{K}_{M / k} / K\right)
$$

for any $\sigma, \tau \in G$. Then it follows from Proposition 1 that $\varphi_{M / K / k}$ induces a surjective homomorphism of $\Lambda(G)$ to $G\left(\hat{K}_{M / k} / K_{M / k}^{*}\right)$ via Artin's resiprocity $\operatorname{map}$ for $H\left(K_{M / k}^{*} / K\right) H /\left(\hat{K}_{M / k} / K\right) \cong G\left(\hat{K}_{M / k} / K_{M / k}^{*}\right)$.

When $\sigma=((K / k) / \mathfrak{a})$ and $\tau=((K / k) / \mathfrak{b})$ for $\mathfrak{a}, \mathfrak{b} \in J_{k}$, we set

$$
\varphi_{M / K / k}(\mathfrak{a} \wedge \mathfrak{b})=\varphi_{M / K / k}(\sigma \wedge \tau) .
$$

Then $\varphi_{M / K / k}$ induces a homomorphism of $\Lambda\left(J_{k}\right)$ to $H\left(K_{M / k}^{*} / K\right) / H\left(\hat{K}_{M / k} / K\right)$.

For the sake of simplicity, we shall use the following notation in general: Suppose that $H, H_{1}$ and $H_{2}$ are subgroups of an abelian group $G$, and $H$ contains both $H_{1}$ and $H_{2}$. Then by the congruence $\alpha \equiv \beta \bmod H$ for $\alpha \in G / H_{1}$ and $\beta \in G / H_{2}$, we mean $a \equiv b \bmod H$, where $a$ and $b$ are representatives of $\alpha$ and $\beta$ in $G$ respectively.

Proposition 2.

(i) Let $M_{1} \supset M_{2} \supset K \supset k$ be a Galois tower, and $K / k$ be abelian. Then for any $\mathfrak{a}, \mathfrak{b} \in J_{k}$ we have

$$
\varphi_{M_{2} / K / k}(\mathfrak{a} \wedge \mathfrak{b}) \equiv \varphi_{M_{1} / K / k}(\mathfrak{a} \wedge \mathfrak{b}) \quad \bmod H\left(\hat{K}_{M_{2} / k} / K\right) .
$$

(ii) Let $M \supset K_{1} \supset K_{2} \supset k$ be a Galois tower, and suppose that both $K_{1} / k$ and $K_{2} / k$ are abelian. Then for any $\mathfrak{a}, \mathfrak{b} \in J_{k}$ we have

$$
\varphi_{M / K_{2} / k}(\mathfrak{a} \wedge \mathfrak{b}) \equiv N_{K_{1} / K_{2}} \varphi_{M / K_{1} / k}(\mathfrak{a} \wedge \mathfrak{b}) \quad \bmod H\left(\hat{K}_{2} / K_{2}\right)
$$

(iii) Let $M \supset K \supset k_{1} \supset k_{2}$ be a Galois tower, and suppose that $K / k_{2}$ is abelian. Then for any $\mathfrak{a}, \mathfrak{b} \in J_{k_{1}}$, we have 


$$
\varphi_{M / K / k_{1}}(\mathfrak{a} \wedge \mathfrak{b}) \equiv \varphi_{M / K / k_{2}}\left(N_{k_{1} / k_{2}} \mathfrak{a} \wedge N_{k_{1} / k_{2}} \mathfrak{b}\right) \quad \bmod H\left(\hat{K}_{M / k_{2}} / K\right) .
$$

Proof. (i) The assertion is implied immediately from (1), (2) and $H\left(\hat{K}_{M_{1} / k} / K\right) \subset H\left(\hat{K}_{M_{2} / k} / K\right)$.

(ii) For $i=1,2$, denote by $\hat{K}_{i}$ the central class field of $K_{i}$ with respect to $M / k$. Put $G_{i}=\operatorname{Gal}\left(K_{i} / k\right), A_{i}=\operatorname{Gal}\left(\hat{K}_{i} / K_{i}\right), \mathbb{S}_{i}=\operatorname{Gal}\left(\hat{K}_{i} / k\right)$, and let $C_{i}$ be a factor set for $\mathscr{S}_{i} / A_{i} \cong G_{i}$. Let further $U_{\sigma_{1}}$ resp. $V_{\sigma_{2}}$ be representatives of $\sigma_{1} \in G_{1}$ resp. $\sigma_{2} \in G_{2}$ in $\mathscr{S}_{1}$ resp. $\mathscr{B}_{2}$. Put $B=\operatorname{Gal}\left(\hat{K}_{1} / \hat{K}_{2}\right)$ and $D=$ $\mathrm{Gal}\left(K_{1} / K_{2}\right)$, and let $W_{\sigma_{2}}$ be a representative of $\sigma_{2} \in G_{2}$ in $G_{1}$. Then by Remark after Proposition 1 we may suppose that $V_{\sigma_{2}}=\left(U_{W_{\sigma_{2}}} \bmod B\right)$. We estimate the norm residue symbol as follows:

$$
\begin{aligned}
\left(\varphi_{M / K_{1} / k}\left(W_{\sigma_{2}} \wedge W_{\tau_{2}}\right), \hat{K}_{2} / K_{1}\right) & =V_{\sigma_{2}}^{-1} V_{\tau_{2}}^{-1} V_{\sigma_{2}} V_{\tau_{2}} \\
=C_{2}\left(\sigma_{2}, \tau_{2}\right) C_{2}\left(\tau_{2}, \sigma_{2}\right)^{-1} & =\left(\varphi_{M / K_{2} / k}\left(\sigma_{2} \wedge \tau_{2}\right), \hat{K}_{2} / K_{2}\right) .
\end{aligned}
$$

Hence $\varphi_{M / K_{2} / k}\left(\sigma_{2} \wedge \tau_{2}\right) \equiv N_{K_{1} / K_{2}} \varphi_{M / K_{1} / k}\left(W_{\sigma_{2}} \wedge W_{\tau_{2}}\right) \bmod H\left(\hat{K}_{2} / K_{2}\right)$. This implies (ii) by setting $\sigma_{2}=\left(\left(K_{2} / k\right) / \mathfrak{a}\right)$ and $\tau_{2}=\left(\left(K_{2} / k\right) / \mathfrak{b}\right)$.

(iii) For $i=1,2$, put $\hat{K}_{i}=\hat{K}_{M / k_{i}}, G_{i}=\operatorname{Gal}\left(K / k_{i}\right), A_{i}=\operatorname{Gal}\left(\hat{K}_{i} / K\right)$, ¿s $_{i}=\operatorname{Gal}\left(\hat{K}_{i} / k_{i}\right)$ and $B=\operatorname{Gal}\left(\hat{K}_{1} / \hat{K}_{2}\right)$. Let $U_{\sigma_{1}}$ resp. $V_{\sigma_{2}}$ be representatives of $\sigma_{1} \in G_{1}$ resp. $\sigma_{2} \in G_{2}$ in $\mathscr{S}_{1}$ resp. $\mathscr{S}_{2}$. Then we have

$$
\begin{aligned}
& \left(\varphi_{M / K / k_{1}}\left(\sigma_{1} \wedge \tau_{1}\right), \hat{K}_{1} / K\right)=U_{\sigma_{1}}^{-1} U_{\tau_{1}}^{-1} U_{\sigma_{1}} U_{\tau_{1}} \\
& \quad \equiv V_{\sigma_{1}}^{-1} V_{\tau_{1}}^{-1} V_{\sigma_{1}} V_{\tau_{1}} \equiv\left(\varphi_{M / K / k_{2}}\left(\sigma_{1} \wedge \tau_{1}\right), \hat{K}_{2} / K\right) \quad \bmod B .
\end{aligned}
$$

This implies the assertion, since

$$
\sigma_{1}=\left(\frac{K / k_{1}}{\mathfrak{a}}\right)=\left(\frac{K / k_{2}}{N_{k_{1} / k_{2}} \mathfrak{a}}\right) \text { and } \tau_{1}=\left(\frac{K / k_{1}}{\mathfrak{b}}\right)=\left(\frac{K / k_{2}}{N_{k_{1} / k_{2}} \mathfrak{b}}\right)
$$

\section{§ 3. Decomposition of $\Lambda(G)$ and central extensions}

Let $M / K / k$ be a Galois tower, and put $G=\operatorname{Gal}(K / k)$ and $\&=\operatorname{Gal}(M / k)$. Then we have

$$
\left.\operatorname{Gal}\left(\hat{K}_{M / k} / K_{M / k}^{*}\right) \cong H^{-3}(G, Z) / \operatorname{Def}_{\circlearrowleft \rightarrow G} H^{-3}(\circlearrowleft), Z\right) .
$$

For this isomorphism, see for instance Kuz'min [4, § 4] or Razar [7, Proof of Lemma 3, (b)]. We call $M$ to be abundant for $K / k$ when $\mathrm{Gal}\left(\hat{K}_{M / k} / K_{M / k}^{*}\right)$ $\cong H^{-3}(G, Z)$. Then it is known that for any Galois extension $K / k$ there always exists an abelian extension $M / K$ which is abundant for $K / k$.

Proposition 3. Let $M / L / K / k$ be a Galois tower. If $M$ is abundant for $L / k$, then $M$ is also abundant for $K / k$. 
Proof. Put $G=\operatorname{Gal}(L / k), G_{1}=\operatorname{Gal}(K / k)$ and $\mathbb{S}=\mathrm{Gal}(M / k)$. If $M$ is abundant for $L / k$, then $\left.\operatorname{Def}_{\mathscr{G} \rightarrow G} H^{-3}(\circlearrowleft), Z\right)=1$ by (3). Since $\operatorname{Def}_{\circlearrowleft \rightarrow G_{1}}=$ $\operatorname{Def}_{G \rightarrow G_{1}} \circ \operatorname{Def}_{\mathscr{G} \rightarrow G}$, the proposition is proved.

The following Proposition is easily obtained.

Proposition 4. Let $L_{i}$ be a central extension of a Galois extension $K_{i} / k$ for $i=1,2$. Then

(i ) $L_{1} \cap L_{2}$ is a central extension of $K_{1} \cap K_{2} / k$,

(ii) $L_{1} L_{2}$ is a central extension of $K_{1} K_{2} / k$.

We call extensions $K_{1}, \cdots, K_{r}$ over $k$ disjoint when $K_{i} \cap K_{j_{1}} \cdots K_{j_{t}}$ $=k$ for any $i$ and any $j_{s}(s=1, \cdots, t)$ such that $K_{i} \neq K_{j_{s}}$.

Now let $M / K / k$ be as before a Galois tower, and assume that $K / k$ is abelian. Put $G=\operatorname{Gal}(K / k)$, and let

$$
G=G_{1} \times \cdots \times G_{r}
$$

be a decomposition to the direct product by cyclic factors $G_{i}$ of $G$ such that the order of $G_{i}$ is divisible by the order of $G_{j}$ when $i<j$. Denote by $G_{i} \wedge G_{j}$ the subgroup of $\Lambda(G)$ generated by all elements $\sigma \wedge \tau$ such that $\sigma \in G_{i}$ and $\tau \in G_{j}$. Then

$$
G_{i} \wedge G_{j} \cong G_{j} \quad \text { for } i<j,
$$

and

$$
\Lambda(G) \cong \oplus \sum_{i<j}\left(G_{i} \wedge G_{j}\right) \cong \oplus \sum_{i<j} G_{j},
$$

where the sum is taken over all pairs $(i, j)$ satisfying $i<j$ for $i, j=1, \cdots, r$. This corresponds to Lyndon [5, Theorem 6].

Let $K_{i}$ be the subfield of $K$ corresponding to $G / G_{i}$ over $k$, and put $K_{i j}=K_{i} K_{j}$. Hence $\operatorname{Gal}\left(K_{i} / k\right) \cong G_{i}$ and $\operatorname{Gal}\left(K_{i j} / k\right) \cong G_{i} \times G_{j}$.

Proposition 5. Notation being as above, assume that $M$ is abundant for $K / k$. Let $\hat{K}_{i j}$ be the central class field of $K_{i j}$ with respect to $M / k$. Then we have

$$
\begin{aligned}
& \left.\hat{K}_{M / k}=\prod_{i<j} \hat{K}_{i j} \quad \text { (disjoint over } K_{M / k}^{*}\right), \\
& \operatorname{Gal}\left(\hat{K}_{i j} / K_{M / k}^{*}\right) \cong G_{i} \wedge G_{j} \cong G_{j} \cong \operatorname{Gal}\left(K_{j} / k\right) \quad \text { for } i<j .
\end{aligned}
$$

Proof. Put $A=\operatorname{Gal}(M / K), \mathscr{S}=\operatorname{Gal}(M / k)$ and $\mathbb{S}_{i}=\operatorname{Gal}\left(M / \prod_{t \neq i} K_{t}\right)$ for $i=1, \cdots, r$. Since $M$ is abundant for $K / k$, the mapping $\varphi$ defined in 
Proposition 1 gives an isomorphism $\Lambda(G) \cong[\mathbb{\mho}$, \& $] / I_{G} A$. For $i<j$, put

$$
\Lambda_{i j}(G)=\oplus \sum_{\substack{s<t \\(s, t) \neq(i, j)}} G_{s} \wedge G_{t} .
$$

Then by taking account of $\mathbb{S S}_{s} \cdot I_{G} A / I_{G} \cdot A$ to be abelian, we have

$$
\begin{aligned}
\varphi\left(\Lambda_{i j}(G)\right) & =\prod_{\substack{s<t \\
(s, t) \neq(i, j)}}\left[\mathscr{S}_{s}, \mathbb{S}_{t}\right] \cdot I_{G} A / I_{G} A \\
& =\left[\mathbb{S}, \prod_{\substack{s \neq i \\
s \neq j}} \mathbb{S}_{s}\right] / I_{G} A=\left[\mathbb{S}, \mathbb{S}_{i j}\right] / I_{G} A,
\end{aligned}
$$

where $\mathscr{S}_{i j}=\operatorname{Gal}\left(M / K_{i j}\right)$. Hence the intermediate field of $\hat{K}_{M / k} / K_{M / k}^{*}$ corresponding to $\varphi\left(\Lambda_{i j}(G)\right)$ is $\hat{K}_{i j}$, and $\left[\hat{K}_{i j}: K_{M / k}^{*}\right]=\left|G_{i} \wedge G_{j}\right|$. Since the intersection of all $\Lambda_{i j}(G)$ is $\{1\}$ and $\varphi$ is an isomorphism, the intersection of all $\varphi\left(\Lambda_{i j}(G)\right)$ is also $\{1\}$. Hence $\hat{K}_{M / k}=\prod_{i<j} \hat{K}_{i j}$. Disjointness follows from $\left[\hat{K}_{M / k}: K_{M / k}^{*}\right]=\left[\prod_{i<j} \hat{K}_{i j}: K_{M / k}^{*}\right] \leqq \prod_{i<j}\left[\hat{K}_{i j}: K_{M / k}^{*}\right]=\prod_{i<j}\left|G_{i} \wedge G_{j}\right|=\mid \Lambda(G)$ $=\left[\hat{K}_{M / k}: K_{M / k}^{*}\right]$.

\section{§4. Norm residue map $\Psi_{M / K / k}$}

Throughout this section, we assume that $K / k$ is abelian, $M / K / k$ is a Galois tower and $M$ is abundant for $K / k$. Put $G=\operatorname{Gal}(K / k), \hat{K}=\hat{K}_{M / k}$ and $K^{*}=K_{M / k}^{*}$. Then

$$
\operatorname{Gal}\left(\hat{K} / K^{*}\right) \cong H^{-1}\left(G, C_{K}\right) \cong H^{-3}(G, Z) \cong \Lambda(G),
$$

where $C_{K}$ is the idele class group of $K$. In Section 2 we defined the mapping $\varphi_{M / K / k}$ of $\Lambda(G)$ to $J_{K} / H(\hat{K} / K)$. In the present section we shall study the inverse mapping of $\varphi_{M / K / k}$.

Let notation be as in Section 3. It follows from Proposition 5 and (7) that

$$
\begin{aligned}
\operatorname{Gal}\left(\hat{K} \mid K^{*}\right) & \cong \oplus \sum_{i<j} \operatorname{Gal}\left(\hat{K}_{i j} / K^{*}\right) \\
& \cong \oplus \sum_{i<j} \operatorname{Gal}\left(K_{j} / k\right) \cong \Lambda(G) .
\end{aligned}
$$

We denote by $I\left(K^{*} / k\right)$ the group of norms of ideals of $K^{*}$ to $k$ which are relatively prime to the discriminants of $M / k$. Let $\mathfrak{a} \in I\left(K^{*} / k\right)$, and $\mathfrak{U}$ be an ideal of $K^{*}$ such that $\mathfrak{a}=N_{K^{*} / k} \mathfrak{A}$. We define a mapping $\psi$ of $I\left(K^{*} / k\right)$ to $\oplus \sum_{i<j} \operatorname{Gal}\left(\hat{K}_{i j} / K^{*}\right)$ by

$$
\psi(\mathfrak{a})=\oplus \sum_{i<j}\left(\frac{\hat{K}_{i j} / K^{*}}{\mathfrak{U}}\right)
$$


Since $\operatorname{Gal}\left(\hat{K} / K^{*}\right)$ is contained in the center of $\operatorname{Gal}(\hat{K} / k)$, the value of $\psi(\mathfrak{a})$ does not depend on the choice of $\mathfrak{X}$. It follows from (8) and (9) that $\psi$ is a surjective homomorphism.

In order to get the image of $\left(\left(\hat{K}_{i j} / K^{*}\right) / \mathfrak{A}\right)$ by the isomorphism

$$
\operatorname{Gal}\left(\hat{K}_{i j} / K^{*}\right) \cong \operatorname{Gal}\left(K_{j} / k\right),
$$

we use the following proposition which is a special case of [1, Proposition 5.1].

Proposition 6. Let $F / k$ be a cyclic extension with $\mathfrak{g}=\mathrm{Gal}(F / k)$ generated by $\sigma, M \supset L \supset F \supset k$ be a Galois tower, and $L / k$ and $M / F$ be abelian. Then

$$
\operatorname{Gal}\left(\hat{L}_{M / k} / L_{M / k}^{*}\right) \cong \mathfrak{D} / C(\mathfrak{S}(L / F)) \Re \cong \operatorname{Gal}\left(F^{\prime} / F\right),
$$

where $D$ is the ideal class group of $F$ corresponding to $M, \mathfrak{S}(L / F)$ is the congruent ideal group of $F$ corresponding to $L, C\left(S_{\mathcal{C}}(L / F)\right)$ is the subgroup of $\mathfrak{D}$ represented by $\mathfrak{S}(L / F), \Re$ is the group of elements $\mathfrak{c}$ of $\mathfrak{D}$ such that $\mathfrak{c}^{\sigma}=\mathfrak{c}$, and $F^{\prime}$ is the subfield of $L$ over $F$ corresponding to $C(\mathfrak{S}(L / F)) \Omega$.

The above isomorphism $\operatorname{Gal}\left(\hat{L}_{M / k} / L_{M / k}^{*}\right) \cong \operatorname{Gal}\left(F^{\prime} \mid F\right)$ is given by

$$
\left(\frac{\hat{L}_{M / k} / L_{M / k}^{*}}{\mathfrak{U}^{*}}\right) \longrightarrow\left(\frac{F^{\prime} / F}{\mathfrak{B}}\right),
$$

where $\mathfrak{U}^{*}$ is any ideal of $L_{M / k}^{*}$ prime to the conductor of $M / F$ and $\mathfrak{B}$ is an ideal of $F$ such that $\mathfrak{B}^{\sigma-1} \equiv N_{L_{M / k}^{*} / F} \mathfrak{A} * \bmod \mathscr{S}(M / F)$.

We apply the above proposition taking $\hat{K}_{i j}, K_{i j}$ and $K_{i}$ instead of $M, L$ and $F$ respectively. Then $L_{M / k}^{*}$ in the proposition becomes $K_{i j}^{*}$ and $F^{\prime}$ becomes $K_{i j}$, because $\operatorname{Gal}\left(F^{\prime} \mid F\right) \cong G_{j} \cong \operatorname{Gal}\left(K_{i j} / K_{i}\right)$ and $\operatorname{Gal}\left(\hat{K}_{i j} / K_{i j}^{*}\right)=G_{j}$ owing to abundantness of $M$ for $K / k$. For $\mathfrak{a} \in I\left(K^{*} / k\right)$, let $\mathfrak{A}_{i}$ and $\mathfrak{B}_{i}$ be ideals of $K_{i}$ such that $N_{K_{i} / k} \mathfrak{I}_{i}=\mathfrak{a}$ and $\mathfrak{B}_{i}^{a_{i}-1} \equiv \mathfrak{A}_{i} \bmod \mathfrak{S}\left(M / K_{i}\right)$, where $\sigma_{i}$ is a generator of the cyclic group $\operatorname{Gal}\left(K_{i} / k\right)$ and $\mathfrak{S}_{\mathfrak{C}}\left(M / K_{i}\right)$ is the ideal group of $K_{i}$ corresponding to $M$. Let further $\mathfrak{b}_{i}=N_{K_{i} / k} \mathfrak{B}_{i}$. We define a mapping $\Psi_{i j}$ of $I\left(K^{*} / k\right)$ to $G_{j}$ for $i<j$ by

$$
\Psi_{i j}(\mathfrak{a})=\left(\frac{K_{j} / k}{\mathfrak{b}_{i}}\right)
$$

Now Proposition 6 implies immediately the following

TheOREM. Let $K / k$ be an abelian extension with $G=\mathrm{Gal}(K / k)$, and $M$ be a Galois extension over $k$ such that $M$ contains $K$ and abundant for $K / k$. Let $G=G_{1} \times \cdots \times G_{r}, K_{i}, K_{i j}$ and $\hat{K}_{i j}$ be as in Section 3 (4) and 
after that. Let the notation $\hat{K}, K^{*}, I\left(K^{*} / k\right)$ and $\Lambda(G)$ be as above. We define a mapping $\Psi_{M / K / k}$ of $I\left(K^{*} / k\right)$ to $\oplus \sum_{i<j} G_{\jmath} \cong \Lambda(G)$ by

$$
\Psi_{M / K / k}(\mathfrak{a})=\oplus \sum_{i<j} \Psi_{i j}(\mathfrak{a}) \quad \text { for } \mathfrak{a} \in I\left(K^{*} / k\right),
$$

where $\Psi_{i j}$ is the mapping defined by (11). Then $\Psi_{M / K / k}$ is a surjective homomorphism of $I\left(K^{*} / k\right)$ to $\oplus \sum_{i<j} G_{j}$ which is isomorphic to $\Lambda(G)$ and so to $\mathrm{Gal}\left(\hat{K} / K^{*}\right)$.

The mapping $\Psi_{M / K / k}$ is regarded as the inverse of the mapping $\varphi_{M / K / k}$ defined in Section 2. In fact we have the following proposition.

Proposition 7. For $\mathfrak{a} \in J_{K}$ denote by [a] an ideal of $K$ such that $(\mathfrak{a}, \hat{K} / K)=((\hat{K} / K) /[\mathfrak{a}])$. Then other notation being as above, we have

$$
\Psi_{i j}\left(N_{K / k}\left[\varphi_{M / K / k}\left(\sigma_{i} \wedge \sigma_{j}\right)\right]\right)=\sigma_{j} .
$$

Proof. To simplify the notation, we put $\hat{K}=\hat{K}_{M / k}$ and $\varphi=\varphi_{M / K / k}$. Put further $G=\operatorname{Gal}(K / k)$ and $A=\operatorname{Gal}(\hat{K} / K)$. Let $U_{\sigma_{i}}$ resp. $U_{\sigma_{f}}$ be representatives of $\sigma_{i}$ resp. $\sigma_{j}$ in $\operatorname{Gal}(\hat{K} / k)$. Then by (1) in Section 2, we have

$$
\begin{aligned}
\left(\frac{\hat{K} / K}{\left[\varphi\left(\sigma_{i} \wedge \sigma_{j}\right)\right]}\right) & =\left(\frac{\hat{K} / K}{\left[\xi_{K / k}\left(\sigma_{i}, \sigma_{j}\right)\right]}\right)\left(\frac{\hat{K} / K}{\left[\xi_{K / k}\left(\sigma_{j}, \sigma_{i}\right)\right]}\right)^{-1} \\
& \equiv U_{\sigma_{i}}^{-1} U_{\sigma_{j}}^{-1} U_{\sigma_{i}} U_{\sigma_{j}} \equiv U_{\sigma_{j}}^{\sigma_{i}-1} \bmod I_{G} A .
\end{aligned}
$$

Let $\hat{\mathfrak{B}}$ be an ideal of $\hat{K}$ such that $U_{\sigma_{j}}=[(\hat{K} / k) / \hat{\mathfrak{B}}]$, the product of the Frobenius automorphisms for the prime factors of $\hat{\mathfrak{B}}$. Then

$$
\left(\frac{\hat{K} / K}{\left[\varphi\left(\sigma_{i} \wedge \sigma_{j}\right)\right]}\right)=\left[\frac{\hat{K} / k}{\hat{\mathfrak{B}}}\right]^{\sigma_{i-1}}=\left[\frac{\hat{K} / k}{\hat{\mathfrak{B}}^{\sigma_{i}-1}}\right]=\left(\frac{\hat{K} / k}{\mathfrak{B}^{\sigma_{i}-1}}\right),
$$

where $\mathfrak{B}=N_{\hat{R} / K} \hat{\mathfrak{B}}$. Let $\mathfrak{B}_{i}=N_{K / K_{i}} \mathfrak{B}$. Then

$$
N_{K / K_{i}}\left[\varphi\left(\sigma_{i} \wedge \sigma_{j}\right)\right] \equiv \mathfrak{B}_{i}^{\sigma_{i}-1} \quad \bmod \mathfrak{S}_{\mathfrak{c}}\left(M / K_{i}\right),
$$

where $\mathfrak{S}_{\mathrm{C}}\left(M / K_{i}\right)$ is, as in Proposition 6, the congruent ideal group of $K_{i}$ corresponding to $M$. Now let $\mathfrak{a}=N_{K / k}\left[\varphi\left(\sigma_{i} \wedge \sigma_{j}\right)\right]$ and $\mathfrak{b}=N_{K_{i} / k} \mathfrak{B}_{i}=N_{\mathbb{R} / k} \hat{\mathfrak{B}}$. Then we have $\Psi_{i j}(\mathfrak{a})=\left(\left(K_{j} / k\right) / \mathfrak{b}\right)=\sigma_{j}$ by (11). Thus the proposition is proved.

\section{REFERENCES}

[ 1 ] Y. Furuta, Note on class number factors and prime decompositions, Nagoya Math. J., 66 (1977), 167-182. 
[2] - A prime decomposition symbol for a non-abelian central extension which is abelian over a bicyclic biquadratic field, Nagoya Math. J., 79 (1980), 79-109.

[ 3 ] F.-P. Heider, Strahlkonten und Geschlechterkörper $\bmod m$, J. reine angew. Math., $320(1980), 52-67$.

[4] L. V. Kuz'min, Homology of profinite groups, Schur multipliers, and class field theory, Math. USSR-Izv., 3 (1969), 1149-1181.

[5] R. G. Lyndon, The cohomology theory of group extensions, Duke Math. J., 15 (1948), 271-292.

[ 6 ] K. Miyake, Central extensions and Schur's multiplicators of Galois groups, Nagoya Math. J., 90 (1983), 137-144.

[ 7 ] M. Razar, Central and genus class fields and the Hasse norm theorem, Cornpositio Math., 35 (1977), 281-298.

Department of Mathematics

Kanazawa University

Marunouchi, Kanazawa 920

Japan 